\title{
Antigenemia y reacción de polimerasa en cadena en tiempo real en el diagnóstico de enfermedad por citomegalovirus en adultos con virus de inmunodeficiencia adquirida
}

\author{
Vivian Luchsinger, Patricia Vásquez, Matías Silva, M. José Bruno, Izkia Siches, \\ Javier Villarroel, M. Luisa Garmendia y Carmen Larrañaga
}

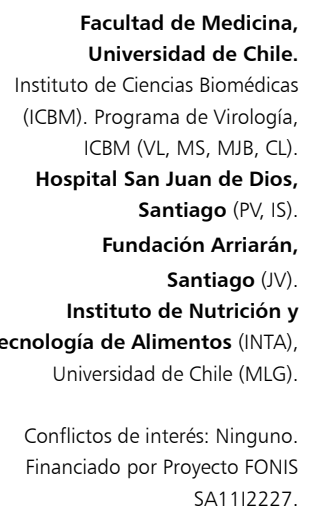

Recibido: 29 de diciembre de 2014

Aceptado: 8 de octubre de 2015

Correspondencia a: Vivian Luchsinger Farías vluchsin@med.uchile.cl

\section{Introducción}

$\mathrm{M}$ ás de $90 \%$ de los adultos chilenos portadores del virus de inmunodeficiencia humana (VIH) está infectado con citomegalovirus $(\mathrm{CMV})^{1}$. Este herpesvirus persiste latente de por vida en el individuo, pudiendo reactivarse, en especial cuando la respuesta inmune celular está disminuida, por lo que constituye una complicación infecciosa y de muerte frecuente en pacientes con síndrome de inmunodeficiencia adquirida $(\mathrm{SIDA})^{2,3}$, aún en la época de terapia antirretroviral $(\mathrm{TARV})^{4-6}$.

Las enfermedades citomegálicas más graves se desarrollan en pacientes leucopénicos (con menos de 50-100 linfocitos $\mathrm{T}$ helper $\left.\mathrm{CD} 4+/ \mathrm{mm}^{3}\right)^{2-7}$, entre las cuales se incluye la retinitis y la enfermedad gastrointestinal (EGI). La primera es la manifestación única más común, representa $85 \%$ de los casos de enfermedad por $\mathrm{CMV}^{2,3,5-8}$ y es indicadora de SIDA $^{8}$. La EGI es la segunda en frecuencia, representando $15 \%$ de las enfermedades por $\mathrm{CMV}$, y puede afectar todo el tracto digestivo ${ }^{5,6}$. La neumonía es menos frecuente y el cuadro clínico es inespecífico ${ }^{5,6}$.

Ganciclovir es el antiviral de elección para CMV y por su alta toxicidad y costo, su aplicación requiere el diagnóstico específico de enfermedad citomegálica. Sólo las técnicas de laboratorio que demuestran infección activa pueden confirmar la enfermedad, incluyendo el aislamiento viral rápido (AVR), la antigenemia o la cuantificación de la carga viral en una reacción de la polimerasa en cadena en tiempo real (RPC-TR $)^{2,3,9}$. El AVR combina el cultivo celular y la detección de antígenos virales por inmunofluorescencia (IF) alcanzando una especificidad superior a $99 \%{ }^{2,9}$. Aunque es el método de referencia ${ }^{2,9}$, su sensibilidad es baja y varía según el paciente, el tipo y condiciones de la muestra estudiada ${ }^{9}$, pudiendo llegar hasta 3\% en sangre. La escasez de laboratorios nacionales con cultivos celulares y la necesidad de preservar la capacidad del virus de infectar mediante el transporte de la muestra rápidamente y en frío al laboratorio limitan su uso.

La técnica más utilizada en Chile para el diagnóstico de enfermedad por CMV es la antigenemia, método rápido que detecta la proteína pp65 del tegumento viral en leucocitos polimorfonucleares (LPMN) de sangre periférica mediante IF indirecta ${ }^{3}$. Su interpretación depende del observador y su positividad disminuye cuando la muestra de sangre es procesada después de cinco horas desde su obtención y en pacientes leucopénicos $(<100$ LT CD4/ $\left.\mathrm{mm}^{3}\right)^{4,9,10}$, lo que ocurre en $45 \%$ de los pacientes chilenos 
infectados por VIH, sin TARV, y en $11 \%$ de los tratados con TARV ${ }^{11}$. La sensibilidad (33-100\%), los valores predictores positivo y negativo (26-100\%) y los valores umbrales $\left(2-13\right.$ céls positivas/2 x $\left.10^{5}\right)$ utilizados para predecir una enfermedad por CMV e indicar terapia antiviral $^{9,10,12,13}$ varían en los distintos estudios internacionales y se desconocen en pacientes chilenos portadores de VIH.

La RPC-TR cuantitativa es una técnica rápida; su sensibilidad y especificidad varían según la condición del paciente y la técnica de referencia que se utilice, entre $61-100 \%$ y $80-99 \%$, respectivamente ${ }^{4,10,12,14}$. Altas cargas sistémicas de CMV en portadores de $\mathrm{VIH}^{3,2,15}$ se han asociado con enfermedad; sin embargo, aún no se ha establecido un valor de corte predictor universal, variando entre 2.645 y $1,6 \times 10^{5}$ copias/ml según el protocolo aplicado, el tipo de muestra y el paciente estudiado ${ }^{5,10,13,15}$.

A diferencia de la antigenemia, la RPC-TR requiere un menor volumen de sangre $(100 \mu \mathrm{L}$ vs $5 \mathrm{ml}$ para la antigenemia), el resultado se obtiene en menor tiempo y es menos dependiente del recuento leucocitario de los pacientes, de las condiciones de obtención y transporte de la muestra y de la subjetividad de quien interpreta los resultados. Sin embargo, su costo es mayor y en muchos laboratorios nacionales aún no se dispone del equipamiento requerido.

En nuestro conocimiento, en nuestro país sólo se han comparado ambas técnicas en receptores de trasplantes, con resultados disímiles y en algunos pacientes incluso discordantes ${ }^{16-18}$, lo que también ha sido reportado en el exterior en enfermos con infección por $\mathrm{VIH}^{13}$.

Aunque la infección por VIH está incorporada en el plan estatal de Garantías Explícitas de Salud-GES ${ }^{8}$, y que la mayoría de los 40.000 chilenos estimados como portadores del VIH está infectado con CMV, las guías clínicas nacionales no incluyen métodos diagnósticos de enfermedad citomegálica. Como se desconoce la utilidad de la antigenemia y de la RPC-TR en el diagnóstico de esta enfermedad, el objetivo de este estudio fue determinar el rendimiento de ambas técnicas y sus valores umbrales predictores de enfermedad por CMV en adultos chilenos con infección por VIH.

\section{Material y Métodos}

\section{Diseño del estudio}

Transversal, de evaluación de pruebas diagnósticas.

\section{Población estudiada}

Ciento setenta y nueve portadores de VIH, mayores de 18 años, atendidos entre marzo de 2012 y septiembre de 2013 en el Hospital San Juan de Dios y en la Fundación Arriarán, seleccionados aleatoriamente. Se incluyeron 77 de quienes acudieron a un control rutinario y no presen- taban cuadro clínico sugerente de CMV (denominados asintomáticos) y 102 adultos hospitalizados o ambulatorios con sospecha clínica de enfermedad por CMV (referidos como sintomáticos); estos casos correspondían a síndrome febril de etiología no precisada, enfermedad gastrointestinal o hepatitis; retinitis y/o neumonía ${ }^{19}$. Por cada paciente se completó una ficha con los antecedentes de edad, género, recuento de LT CD4, TARV, tiempo de diagnóstico y carga plasmática de VIH, y de los enfermos se registraron antecedentes clínicos del episodio en curso (sintomatología, signología, parámetros de laboratorio, evolución clínica, diagnóstico etiológico y terapia con ganciclovir).

\section{Muestras}

De todos los individuos se obtuvo $4 \mathrm{ml}$ de sangre venosa periférica que fue depositada en un tubo con EDTA y transportada de inmediato tras su obtención al laboratorio en hielo.

\section{Técnicas de laboratorio}

Se realizó aislamiento viral rápido (AVR), antigenemia y RPC-TR cuantitativa en paralelo a cada muestra.

AVR: $0,2 \mathrm{ml}$ de sangre se inocularon en un vial con células MRC-5, centrifugándose por 15 min a 2.490 g en centrifuga U-32R Boeco ${ }^{\circledR}$. Tras 24 h de incubación con 1 $\mathrm{ml}$ de medio MEM y $1 \%$ de suero fetal de bovino, a $37^{\circ} \mathrm{C}$ con $\mathrm{CO}_{2}$, se fijó con $1 \mathrm{ml}$ de metanol por $5 \mathrm{~min}$ y un $1 \mathrm{ml}$ de acetona por $10 \mathrm{~min}$ a temperatura ambiente. La detección de antígenos virales se realizó por inmunofluorescencia indirecta (IFI) con el kit Light Diagnostics ${ }^{\mathrm{TM}}$, según instrucciones del fabricante. Los preparados se observaron al microscopio de fluorescencia Nikon $\AA$ con un aumento de 20X. La observación de $\geq 1$ núcleo fluorescente se informó como muestra positiva.

Antigenemia: Se separaron los leucocitos con dextrán y se detectó la proteína pp65 mediante IFI con CINAkit (Argene $\left.{ }^{\circledR}\right)$, según instrucciones del productor. Por cada paciente se observaron 400.000 céls a $40 \mathrm{X}$ en un microscopio Nikon ${ }^{\circledR}$. La muestra se consideró positiva con la presencia de $\geq 1$ núcleo polilobulado fluorescente y los resultados se informaron como recuento de céls positivas/400.000 observadas.

$R P C$-TR cuantitativa: En $40 \mu \mathrm{L}$ de la fracción de leucocitos utilizada para la antigenemia (concentración de $2 \mathrm{x}$ $10^{6}$ céls $/ \mathrm{ml}$ ), se realizó la extracción de ADN mediante el kit High Pure Viral NucleicAcid Kit $\left(\right.$ Roche $\left.^{\circledR}\right)$, de acuerdo a las instrucciones del proveedor. El ADN extraído fue almacenado a $-80^{\circ} \mathrm{C}$ y para su posterior análisis se agregaron $2 \mu \mathrm{l}$ a $18 \mu \mathrm{l}$ de la mezcla formada por $4 \mu \mathrm{L}$ de Master HibridizationProbe $\left(\right.$ Roche $\left.^{\circledR}\right) ; 0,7 \mu \mathrm{M}$ de cada partidor (gB-F y gB-R); 0,25 $\mu \mathrm{M}$ de cada sonda (gB-FL y gB-LC) ${ }^{14} ; 0,5 \mu \mathrm{L}$ de uracil ADN glicosilasa y $9,7 \mu \mathrm{L}$ de agua estéril. En un termociclador Light Cycler $2.0^{\circledR} \mathrm{se}$ 
amplificó un fragmento del gen de la glicoproteína B de $\mathrm{CMV}$ incubando a $10^{\circ} \mathrm{C}$ por $10 \mathrm{~min}$, a $95^{\circ} \mathrm{C}$ por $10 \mathrm{~min}$ y con 45 ciclos de $10 \mathrm{seg}$ a $95^{\circ} \mathrm{C}, 15 \mathrm{seg}$ a $58^{\circ} \mathrm{C}$ y $12 \mathrm{seg}$ a $72^{\circ} \mathrm{C}$; seguidos por $1 \mathrm{~min}$ a $50^{\circ} \mathrm{C}$ y $2 \min$ a $40^{\circ} \mathrm{C}^{14}$. En cada corrida se incluyó un control negativo y como control positivo el plásmido pCMV- KS- T 3214 bp (Roche®). Para estimar el número de copias de ADN de CMV de cada muestra se construyó una curva con concentraciones del plásmido de $1 \times 10^{2} ; 1 \times 10^{3} ; 1 \times 10^{4} ; 1 \times 10^{5} ; 1 \times 10^{6}, 1 \times$ $10^{7}$ y 1 x $10^{8}$ copias de ADN/ $\mu \mathrm{L}$. El umbral de detección fue de 25 copias/ $\mu \mathrm{L}$. Para los análisis cuantitativos se excluyeron las muestras con $<100$ copias $/ \mu \mathrm{L}$. Se repitió la amplificación en diluciones de las muestras con valores superiores a la concentración máxima utilizada y posteriormente se calculó la concentración ajustando por el factor de dilución utilizado. Los resultados obtenidos de cada muestra se expresaron como $\log _{10}$ copias de ADN de $\mathrm{CMV} / 2$ x $10^{6}$ céls.

\section{Análisis estadístico}

Se describieron las variables de interés a través de estadísticas descriptivas (medianas, porcentajes) y de dispersión (rango intercuartílico), según correspondiera. Se compararon diferencias entre pacientes, con y sin sospecha de CMV, mediante las pruebas $\chi^{2}$ y exacta de

Fisher para las variables cualitativas y $\mathrm{t}$ de Student con corrección de Welch, Kruskal-Wallis y Mann-Whitney para las cuantitativas. En base a tablas de contingencia 2 × 2, se determinó la sensibilidad, especificidad y los valores predictores positivos (VPP) y negativos (VPN). Se establecieron los puntos de corte, según los valores de sensibilidad y especificidad más altos, y el área bajo la curva de la RPC-TR respecto a la antigenemia. La concordancia entre ambas técnicas se determinó mediante el coeficiente каppa e intervalos de confianza a $95 \%$. De acuerdo a lo publicado ${ }^{20}$, se consideró concordancia leve con $\kappa<0,2$; aceptable con $\kappa$ entre $0,21-0,4$; moderada entre $0,41-0,6$; considerable entre $0,61-0,8$ y casi perfecta si $\kappa \geq 0,81$. La correlación entre los núcleos positivos de la antigenemia y las copias de ADN de CMV determinadas por la RPC-TR se determinó por la prueba de Spearman. Para los análisis estadísticos se utilizó el programa Stata 11.0 considerando significativo un valor de $\mathrm{p}<0,05$.

Aspectos éticos: Todos los pacientes aceptaron participar en el estudio voluntariamente a través de la firma de un consentimiento informado. El proyecto fue aprobado por los Comités de Ética de Investigación en Seres Humanos del Servicio de Salud Metropolitano Occidente y Central y de la Facultad de Medicina, Universidad de Chile.

\section{Resultados}

Tabla 1. Características demográficas y clínicas de 179 adultos infectados con VIH, con y sin sospecha de enfermedad por CMV

\begin{tabular}{|c|c|c|c|}
\hline Características* & $\begin{array}{l}\text { Con sospecha de } \\
\text { enfermedad por } \\
\text { CMV } \\
n=102\end{array}$ & $\begin{array}{l}\text { Sin sospecha de } \\
\text { enfermedad por } \\
\text { CMV } \\
n=77\end{array}$ & Valor $p$ \\
\hline Edad: mediana (RICp25-p75) & $36(30-56)$ & $34(21-58)$ & 0,1 \\
\hline Género: masculino n (\%) & $81(79,4 \%)$ & $53(68,8 \%)$ & 0,07 \\
\hline $\begin{array}{l}\text { Procedencia } \\
\text { Hospital San Juan de Dios } \\
\text { Fundación Arriarán }\end{array}$ & $\begin{array}{l}68(66,6 \%) \\
34(33,3 \%)\end{array}$ & $\begin{array}{l}64(83,1 \%) \\
13(16,9 \%)\end{array}$ & 0,008 \\
\hline $\begin{array}{l}\text { Diagnóstico de infección por VIH } \\
\text { Mediana (RICp25-p75) }\end{array}$ & $24,5(1,75-156)$ & $15(2-118)$ & 0,1 \\
\hline $\begin{array}{l}\text { Carga plasmática de VIH } \\
\text { Copias x 103/ml } \\
\text { Mediana (RICp25-p75) }\end{array}$ & $120(22-2.650)$ & $9,9(46-485)$ & $<0,001$ \\
\hline $\begin{array}{l}\text { Recuento LT CD4 céls./ml } \\
\text { Mediana (RICp25-p75) }\end{array}$ & $40(10-270)$ & $182(85-379)$ & $<0,001$ \\
\hline $\begin{array}{l}\text { \% LT CD4 } \\
\text { Mediana (RICp25-95) }\end{array}$ & $6(3-22)$ & $13(8,5-26,3)$ & $<0,001$ \\
\hline $\begin{array}{l}\text { Con terapia antirretroviral, n (\%) } \\
\text { Terapia antirretroviral (meses) } \\
\text { Mediana (RICp25-p75) }\end{array}$ & $43(42,2 \%)$ & $54(70,1 \%)$ & $<0,001$ \\
\hline
\end{tabular}

\section{Población estudiada}

Los 102 adultos portadores de VIH con sospecha clínica de enfermedad por CMV y los 77 casos sin cuadro clínico sólo se diferenciaron en la carga plasmática de VIH, el recuento de LT CD4 y la proporción con TARV (Tabla 1), siendo similar el recuento de leucocitos sanguíneos establecido en la fracción utilizada para la antigenemia (mediana en los dos grupos $=8,3 \times 10^{3} / \mu \mathrm{L}$; rangos: $1,5-43,2 \times 10^{3} / \mu \mathrm{L}$ y $2,4-47,8 \times 10^{3} / \mu \mathrm{L}$, respectivamente; $p>0,05)$.

Entre los 102 enfermos, $66(63,5 \%)$ estaban hospitalizados, con una mediana de 13 días de hospitalización (RIC: 7-60) y de 30 días de evolución de la enfermedad (RIC: 14-180). La enfermedad gastrointestinal o hepatitis fue el diagnóstico más frecuente con 56 casos $(54,9 \%)$, seguido de $35(34,3 \%)$ con neumonía, 18 (17,6\%) con síndrome febril, $9(8,7 \%)$ con retinitis, uno con shock séptico $(0,95 \%)$ y uno con úlcera perianal $(0,95 \%)$. El seguimiento clínico a la semana pos enrolamiento fue posible en 82 de los pacientes (80,4\%): 13 de ellos $(15,5 \%)$ estaba recibiendo terapia con ganciclovir (GCV), 27 estaban asintomáticos $(32,9 \%)$ y dos habían fallecido $(2,4 \%)$. Los $57(74,0 \%)$ adultos sin enfermedad por CMV controlados a la semana permanecían asintomáticos $\mathrm{y}$ ninguno había recibido GCV. 


\section{Detección de CMV}

En los 179 casos totales estudiados, la antigenemia fue positiva en $49(27,4 \%)$, la RPC-TR en $41(22,9 \%)$ (p: 0,3) y en seis $(3,4 \%)$ se aisló CMV en sangre $(p<0,0001)$. Estos seis pacientes eran hombres entre 31 y 53 años de edad (mediana: 52 años) y sintomáticos: uno con retinitis; otro con neumonía y perforación intestinal con biopsia sugerente de infección por CMV y los otros cuatro con diarrea, tres de los cuales además tenían un síndrome febril. Sólo dos recibieron ganciclovir y otros dos fallecieron; cuatro estaban hospitalizados y cuatro recibían TARV. Las cargas plasmáticas de VIH variaron entre 53 y 1.600 .000 copias $/ \mathrm{mL}$ (mediana: 8.400 copias $/ \mathrm{mL}$ ) y los recuentos de LTCD4 entre 9 y 61 céls $/ \mathrm{mm}^{3}$ (mediana: 18). En todos ellos fueron positivas la antigenemia, con una mediana de 64,5 núcleos/400.000 céls observadas (rango: 4-338), y la RPC-TR, con una mediana de 5,4 $\log _{10}$ copias de ADN de CMV/2 x $10^{6}$ células (rango: 4,5 y 6,8).

\section{Comparación de la antigenemia y la RPC-TR}

En 157/179 adultos estudiados (88\%), fue concordante el resultado entre la antigenemia y la RPC-TR (coeficiente $\kappa=0,67$; IC 95\%: 0,54-0,79). En base a la detección en enfermos y no enfermos, la sensibilidad, especificidad, el VPP y VPN de la antigenemia fue 46\%, 97\%, 96\% y 58\% y de la RPC-TR 36\%, 95\%, 90\% y 53\%, respectivamente.

Utilizando la antigenemia como referencia, la sensibilidad de la RPC-TR fue 71\%, la especificidad 95\%, el VPP $83 \%$, el VPN $89 \%$ y la exactitud $88 \%$. Por la ausencia de una técnica de referencia apropiada, estos parámetros se determinaron respecto a un estándar ampliado definido como paciente con sospecha clínica de enfermedad por CMV y una de las técnicas positivas, aumentando la sensibilidad y el VPN de la antigenemia a 92 y $95 \%$ y de la RPC-TR a $72 \%$ y $80 \%$, respectivamente. La especificidad de la antigenemia disminuyó a $80 \%$ y el VPP a $72 \%$, mientras la RPC-TR mantuvo su especificidad y el VPP aumentó levemente a $92 \%$.

El área bajo la curva ROC (AUC) de la antigenemia fue 0,71 (IC 95\%: 0,64-0,77) respecto a todos los casos sintomáticos y de 0,90 (IC 95\%: 0,85-0,94) considerando los enfermos con RPC-TR positiva. El valor de corte fue $\geq 1$ núcleo positivo, con $46,1 \%$ de sensibilidad; $97,4 \%$ de especificidad; 17,7 de LR (+); 0,55 de LR (-) y 68,2\% de clasificación correcta. El AUC de la RPC-TR fue regular, con un valor de 0,65 (IC 95\%: 0,58-0,72) respecto a todos los enfermos y de 0,83 (IC 95\%: 0,77-0,88) si se analizan los enfermos con antigenemia positiva. El valor de corte fue $\geq 5,5 \log _{10}$ copias $/ 2$ x $10^{6}$ céls (LR+: 2,27 y LR-: 0,42).

El coeficiente de correlación de Spearman entre las copias de ADN de CMV determinada por la RPC-TR y el número de núcleos positivos en la antigenemia de adultos con infección por VIH fue 0,36 (p: 0,03) (Figura 1). Las copias de CMV en los portadores de VIH con antige- nemia positiva fueron similares a las de los pacientes con antigenemia negativa, con una mediana de 5,8 y 4,7 (RIC: 4,6-6,5 y 3,9-5,9 $\log _{10}$ copias de ADN de CMV/2 x $10^{6}$ céls, respectivamente) (prueba de Mann-Whitney, $\mathrm{p}=0,1)$ (Figura 2).

Tanto la positividad de la antigenemia como de la RPC-TR fue mayor en los sintomáticos que en los asintomáticos, siendo $47(46,1 \%)$ y $37(36,3 \%)$ en los 102 enfermos y $2(2,6 \%)$ y $4(5,2 \%)$ en los 77 no enfermos, respectivamente $(\mathrm{p}<0,0001)$. En ambos grupos de adultos, la detección por las dos técnicas fue similar $(p \geq 0,2)$. La antigenemia tuvo una mediana de 10 núcleos

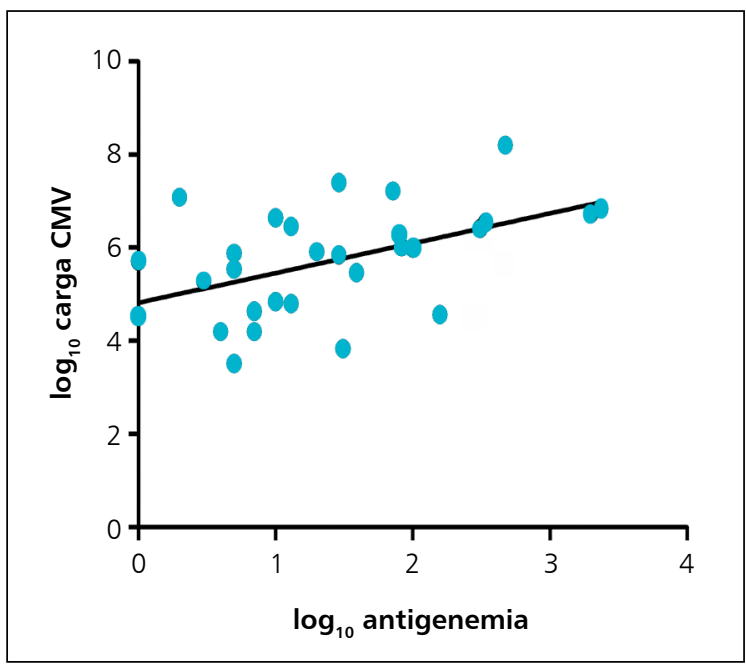

Figura 1. Correlación de copias de ADN de CMV por RPC-TR en leucocitos y núcleos positivos en antigenemia en 30 adultos con infección por VIH. Coeficiente de Spearman $=0,36$ $(p=0,03)$.

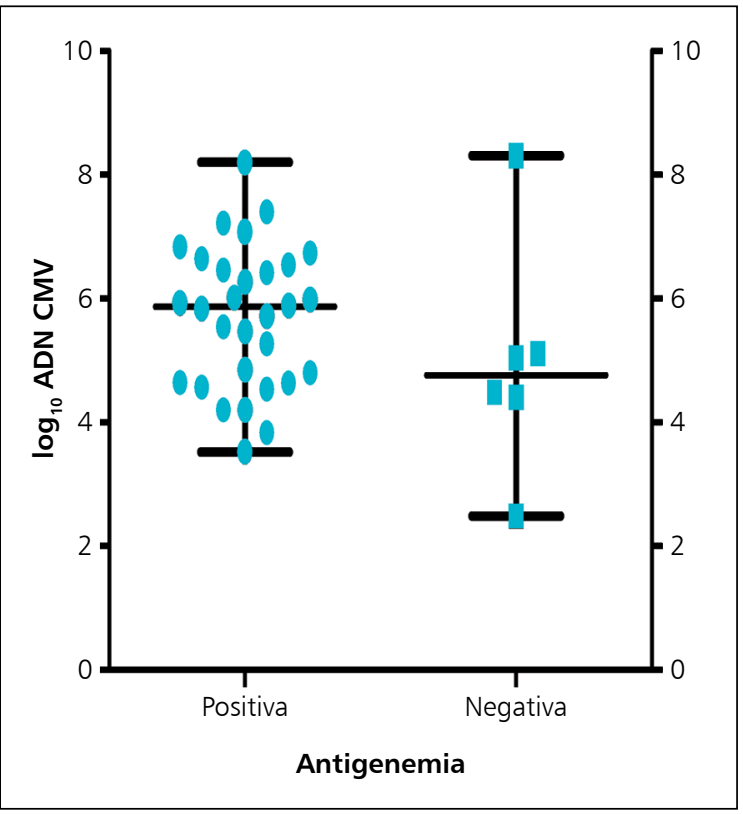

Figura 2. Copias de ADN de CMV en leucocitos de adultos con infección por $\mathrm{VIH}$, con y sin antigenemia positiva. Líneas horizontales representan las medianas $p=0,4$ con prueba de Mann-Whitney. 
positivos/400.000 céls analizadas en los enfermos (rango: 4-80) y sólo se observó un núcleo fluorescente en cada asintomático. Las cargas de CMV detectadas por RPC-TR no fueron significativamente diferentes entre los pacientes (mediana: 6,7; rango: 4,6-66,7 $\log _{10}$ copias de ADN de $\mathrm{CMV} / 2 \times 10^{6}$ céls) y los asintomáticos (mediana: 5,1; rango: 4,1-5,3) (prueba Mann-Whitney, p: 0,6).

Entre los enfermos, la sensibilidad, especificidad, VPP y VPN de la RPC-TR respecto a la antigenemia fueron $72 \%, 95 \%, 92 \%$ y $80 \%$ (Tabla 2 ) y el área bajo la curva ROC 0,83 (IC 95\%: 0,77-0,88). Considerando los enfermos con ambas técnicas positivas, el AUC de la antigenemia fue 0,84 (IC 95\%: 0,78-0,89) y de la RPC-TR de 0,91 (IC 95\%: 0,86-0,95).

Comparando los 47 enfermos con antigenemia positiva y los 55 con antigenemia negativa, la RPC-TR amplificó CMV en $35(72,3 \%)$ y $3(5,5 \%)$, respectivamente $(\mathrm{p}<0,0001)$. En los primeros, los recuentos de LT CD4 fueron menores (mediana: 37 vs 49,5; rangos: 8,7-60,2 y 12,2-125,5 céls/ml; prueba t, p: 0,02). Ambos grupos tuvieron similares recuentos leucocitarios (medianas: 7,9 vs 8,3 ; rangos: $5,6-11,3$ y $5,0-12,3 \times 10^{3}$ leucocitos/ $\mu 1$ ), cargas plasmáticas de VIH (medianas: 98 vs 94; rangos: 26,5-380 y 40-460) y de CMV (medianas: 4,8 vs 4,7; rangos: 4,8-6,7 y 3,8-5,6 $\log _{10}$ copias de ADN de CMV/2 x $10^{6}$ céls $)(p>0,1$, prueba $t)$. Síndrome febril fue el diag-

\section{Tabla 2. Detección de CMV mediante antigenemia y RPC-TR en 179 adultos} con infección por VIH

\begin{tabular}{lrrrrr} 
Casos & $(\mathbf{n})$ & $+\mathbf{r}$ & \multicolumn{2}{c}{ Antigenemia/RPC-TR } \\
& & $+/-$ & $-/+$ & $-/-$ \\
Enfermos & $(102)$ & 34 & 13 & 3 & 52 \\
Asintomáticos & $(77)$ & 0 & 2 & 4 & 71 \\
Total & $(179)$ & 34 & 15 & 7 & 123 \\
\hline
\end{tabular}

nóstico clínico en $10(21,3 \%)$ pacientes con antigenemia positiva y en $6(10,9 \%)$ de los negativos; neumonía en $17(36,2 \%)$ y $19(34,5 \%)$; enfermedad gastrointestinal y/o hepatitis en $25(53,2 \%)$ y $30(54,5 \%)$ y retinitis en 3 $(6,4 \%)$ y $6(10,5 \%)$, respectivamente (prueba de Fisher, $\mathrm{p}>0,4)$.

Los 37 enfermos con RPC-TR positiva y los 65 con RPC-TR negativa tuvieron similar edad, carga plasmática de $\mathrm{VIH}$, recuento de leucocitos sanguíneos (mediana: $8,3 \times 10^{3}$ vs $8,4 \times 10^{3}$ céls/ $\mu L$; RIC: $5,4-12,9$ y $5,8-12,3$ $\mathrm{x} 10^{3}$ céls $\left./ \mu \mathrm{L}\right)$, número de núcleos fluorescentes en la antigenemia $(p>0,1)$ y diagnósticos clínicos $(p>0,1)$ con síndrome febril en 9 casos de cada grupo $(24,3 \%$ y $13,9 \%)$; neumonía en 15 (40,5\%) y 21 (32,3\%); compromiso GI en $20(54,1 \%)$ y $21(53,9 \%)$ y retinitis en dos $(5,5 \%)$ y $7(10,8 \%)$. Ambos grupos sólo se diferenciaron significativamente en el recuento de LT CD4: mediana: 21 y 54,0 céls $/ \mathrm{ml}$, respectivamente $(\mathrm{p}=0,004$ por prueba $\mathrm{t})$.

La positividad de la antigenemia y de la RPC-TR $(p>0,1)$ no varió entre los pacientes con distinto cuadro clínico, siendo en ambas técnicas menor entre los pacientes con retinitis y mayor entre quienes presentaron un síndrome febril $(\mathrm{p}>0,1)$ (Tabla 3). Los recuentos de leucocitos, de LT CD4, de cargas plasmáticas de VIH (datos no mostrados) y de CMV fueron similares entre los enfermos con distinto diagnóstico (ANOVA, $p>0,1$ ) (Tabla 3).

\section{Discusión}

Para diagnosticar una enfermedad por CMV se dispone de diversos métodos, cuya utilidad en la práctica clínica debe ser establecida en las diferentes poblaciones de inmunocomprometidos. En este estudio de 179 adultos portadores del VIH, la positividad de la antigenemia y de la RPC en tiempo real para el diagnóstico de enfermedad

Tabla 3. Detección de CMV según técnica y diagnóstico clínico en 102 adultos con infección por VIH con sospecha clínica de enfermedad por CMV

\begin{tabular}{|c|c|c|c|c|c|}
\hline \multirow[t]{2}{*}{ Cuadro clínico } & \multicolumn{2}{|c|}{ Antigenemia (+) } & \multicolumn{2}{|r|}{ RPC-TR (+) } & \multirow[t]{2}{*}{$p^{*}$} \\
\hline & $\begin{array}{l}\text { Casos } \\
\text { n (\%) }\end{array}$ & $\begin{array}{l}\text { Núcleos (+) } \\
\text { Mediana (RIC) }\end{array}$ & $\begin{array}{l}\text { Casos } \\
\text { n (\%) }\end{array}$ & $\begin{array}{c}\log _{10} \text { copias ADN de CMV/2 } \times 10^{6} \text { céls } \\
\text { Mediana (RIC) }\end{array}$ & \\
\hline $\begin{array}{l}\text { Síndrome febril } \\
(\mathrm{n}=18)\end{array}$ & $12(66,6)$ & $1(0-17,5)$ & $9(50,0)$ & $5,3(4,6-6,3)$ & 0,4 \\
\hline $\begin{array}{l}\text { Neumonía } \\
(\mathrm{n}=35)\end{array}$ & $17(48,6)$ & $0(0-11,5)$ & $15(42,9)$ & $6,3(4,8-8,0)$ & 0,8 \\
\hline $\begin{array}{l}\text { Enfermedad gastrointestinal } y / 0 \text { hepatitis } \\
(n=56)\end{array}$ & $24(42,9)$ & $0(0-9,2)$ & $20(35,7)$ & $5,5(4,6-6,5)$ & 0,5 \\
\hline $\begin{array}{l}\text { Retinitis } \\
(\mathrm{n}=9)\end{array}$ & $3(33,3)$ & $4(2-80)$ & $2(22,2)$ & $5,2(4,2-6,2)$ & 1,0 \\
\hline
\end{tabular}


por CMV fue similar por lo que asociado a su menor costo, mayor accesibilidad y número de casos positivos, aunque sin significancia estadística, proponemos a la antigenemia como la primera opción para el diagnóstico de esta enfermedad viral en la población estudiada. La RPC-TR es una alternativa apropiada y, de acuerdo a la buena concordancia entre las dos técnicas, sería de elección en aquellos casos en los cuales no es posible realizar la antigenemia en condiciones óptimas, como la imposibilidad de procesar la muestra de sangre en menos de $5 \mathrm{~h}$.

Por la infrecuente detección de CMV en sangre de adultos con VIH asintomáticos, aún en leucocitos, una antigenemia o RPC positiva plantea al CMV como probable agente etiológico en pacientes con sospecha de enfermedad citomegálica, independiente de la carga viral $^{5}$. Así, con $\geq 1$ núcleo positivo/400.000 céls en la antigenemia, 68,2\% de los enfermos fueron clasificados como tales. Respecto a la RPC-TR, si se considera la carga de ADN de CMV mayor a la máxima detectada en asintomáticos como indicadora de infección activa, en $44 \%$ de los enfermos se habría descartado enfermedad por CMV, pese a que en $88 \%$ de ellos la antigenemia fue $\geq 1$ núcleo positivo. Por las menores cargas de CMV detectadas ${ }^{5,21}$ en este estudio y por su relación más directa con replicación viral, es de interés verificar este valor de corte en plasma con una RPC-TR validada para uso clínico.

Por el alto VPN ( $\geq 95 \%$ ) de la antigenemia detectada en este estudio, coincidente con publicaciones extranje$\operatorname{ras}^{12,22}$, un resultado negativo permitiría descartar a CMV como agente etiológico en un portador de VIH enfermo; por el contrario, dado el alto VPP $(97,6 \%)$ en pacientes con un recuento de $\mathrm{LT}$ CD $4<100$ céls $/ \mu \mathrm{L}$, una antigenemia positiva confirmaría a CMV como agente en los casos con sospecha clínica de esta enfermedad.

Tanto la positividad de la antigenemia como de la RPC-TR detectadas en este estudio se incluyen en el rango de publicaciones extranjeras para el diagnóstico de enfermedad por CMV en portadores de VIH (33-100\% de la antigenemia y 5,2-35\% de la RPC) ${ }^{12,22-27}$. A nivel nacional, nuestros resultados sólo pueden compararse con lo publicado en receptores de trasplantes ${ }^{16-18}$, en quienes la positividad de la antigenemia $(40 \%)$ y de la RPC ( $>32 \%)^{17,18}$ fueron mayores, en especial en pacientes que recibieron un trasplante de órganos sólidos con sospecha clínica de enfermedad (58\% de la antigenemia y $73 \%$ de la RPC) ${ }^{16}$, aunque la concordancia fue moderada $(\kappa=0,57)$ y menor a la de este estudio. Diferencias en los cuadros clínicos, recuentos de LT CD4 y TARV de los pacientes enrolados-factores que inciden en la frecuencia de enfermedad por este virus, técnicas de amplificación y valores umbrales aplicados para definir resultados positivos (entre 900 y $1,6 \times 10^{5}$ copias referidos a $10^{5} \mathrm{o}$ $10^{6}$ céls o LPMN o por ml de LPMN o leucocitos) ${ }^{5,24-28}$ dificultan las comparaciones y enfatiza la necesidad de establecer en nuestro país la positividad de las técnicas disponibles en cada población de inmunocomprometidos, idealmente utilizando RPC universales.

La concordancia aceptable entre la antigenemia y la RPC-TR en los enfermos concuerda con lo publicado a nivel internacional ( $\kappa$ entre 0,4 y 0,9$)^{10,29}$. Por el contrario, en asintomáticos fueron totalmente discordantes y complementarias, puesto que por la antigenemia negativa se podría descartar replicación viral activa en los cuatro casos con RPC positiva. La evolución de la carga viral permitiría definir si este resultado corresponde a ADN viral latente o replicación del virus $s^{2,7,9,10}$.

Una RPC negativa en pacientes con antigenemia positiva -observada en 15 pacientes en este estudio- ha sido previamente reportada ${ }^{13,23}$. Los casos con sólo un núcleo positivo podrían considerarse falsos positivos; algunos de los sin amplificación genómica viral podrían explicarse por la pérdida de ácidos nucleicos de los extractos, por la descongelación desde su almacenamiento a $-80^{\circ} \mathrm{C}, \mathrm{y}$ en otros, la discrepancia se explicaría por una replicación viral local que no se expresa a nivel sistémico, por lo que no aumenta la carga de CMV en la sangre ${ }^{9,10}$. Como se ha demostrado, en estos casos la presencia de la proteína pp65 se debe a la transferencia pasiva desde células endoteliales infectadas a los polimorfonucleares ${ }^{29-32}$. Como en ellas el virus no replica ni establece latencia, no se detecta el genoma viral, siendo éstas las células predominantes en la fracción que se obtiene con dextrán para la antigenemia y en la cual se aplicó la RPC en este estudio.

De acuerdo con lo previamente descrito ${ }^{9}$ y confirmando su inutilidad como técnica de referencia, la positividad del aislamiento viral rápido en sangre fue muy baja. Sin embargo, aislar el virus indicaría enfermedad por CMV dado que todos eran pacientes sintomáticos y tuvieron antigenemia y RPC-TR positivas. Como el virus se aisló en 2/3 lavados bronquio-alveolares de pacientes con neumonía y fue el único método que detectó CMV en uno de ellos, el AVR es útil y recomendable en muestras biológicas obtenidas directamente del órgano blanco afectado $^{3}$. Puesto que esto no será posible en la mayoría de los casos porque involucra procedimientos invasores (LBA, biopsia) de difícil acceso o porque no está definido el foco infeccioso (síndrome febril), se requiere establecer la sensibilidad de técnicas como la antigenemia y la RPCTR en sangre, por ser la muestra que rutinariamente se obtiene en los enfermos.

Una limitación de este estudio es la clasificación de enfermos en base sólo a criterios clínicos. Para disminuir la subjetividad se aplicaron definiciones universales de enfermedad por $\mathrm{CMV}^{19}$ y se uniformaron criterios entre los médicos que enrolaron pacientes, todos con amplia experiencia en atención de enfermos con infección por VIH. Pese a lo anterior, la significativamente superior 
positividad de las dos técnicas en los enfermos respecto a los sin sintomatología demuestra su utilidad en el diagnóstico de enfermedad por CMV en adultos con VIH.

La ausencia de diferencias significativas en la proporción de positivos por antigenemia o RPC-TR respecto a los distintos cuadros clínicos incluidos, debiera ser corroborada con un mayor número de pacientes en cada categoría, para confirmar si la menor positividad de la antigenemia en los pacientes con retinitis en relación a los con síndrome febril corresponde a una sensibilidad de la técnica o del cuadro clínico, puesto que una infección activa en un determinado compartimento, como es el ojo, podría no expresarse a nivel sistémico.

En conclusión, en adultos chilenos portadores de VIH con sospecha clínica de enfermedad por CMV, la positividad de la antigenemia y la RPC-TR cuantitativa para el diagnóstico de esta enfermedad es similar y complementario. Valores $\geq 1$ núcleo $(+) / 400.000$ céls en la antigenemia $\mathrm{y} \geq 5,5 \log _{10}$ de copias de ADN de $\mathrm{CMV} / 2 \times 10^{6}$ leucocitos indicarían enfermedad por CMV. El aislamiento viral rápido en sangre no es una técnica apropiada como estándar debido a su baja positividad.

Agradecimientos. A Cristian Moreno, Dina Silva y Luis Torres por su valioso trabajo técnico; a Dr. Marcelo Wolff y al personal de la Fundación Arriarán y a las matronas Marisol Muñoz y Cecilia Vera del Hospital San Juan de Dios por su esencial colaboración en la toma y envío de muestras.

\section{Resumen}

Introducción: La infección por citomegalovirus (CMV) es frecuente en adultos con virus de inmunodeficiencia humana (VIH). No se ha establecido la utilidad de los métodos cuantitativos para diagnosticar enfermedad por CMV en pacientes chilenos. Objetivo: Determinar la positividad de antigenemia y reacción de polimerasa en cadena en tiempo real (RPC-TR) en el diagnóstico de enfermedad por $\mathrm{CMV}$ en adultos chilenos con infección por VIH. Metodología: Se detectó CMV mediante aislamiento viral rápido (AVR), antigenemia y reacción de polimerasa en cadena en tiempo real (RPCTR) cuantitativa en adultos infectados por VIH, con y sin sospecha de enfermedad por CMV. Resultados: El recuento de LT CD4 fue menor y mayor la carga de VIH en 102 sintomáticos respecto a 77 asintomáticos $(\mathrm{p}<$ $0,05)$. La antigenemia y la RPC-TR fueron positivas en 46 y $36 \%$ de los enfermos y en 3 y 5\% de los asintomáticos respectivamente. La sensibilidad, especificidad, valor predictor positivo y negativo de la antigenemia y la RPC-TR fueron $92 \%, 80 \%, 72 \%$ y $95 \%$ y $72 \%, 95 \%$, $92 \%$ y $80 \%$, respectivamente. Los valores de corte fueron $\geq 1$ núcleo $(+)$ y $\geq 5,5 \log _{10}$ copias $/ 2 \times 10^{6}$ céls. Se aisló CMV en 3,4\%, todos los sintomáticos. Conclusión: La antigenemia y la RPC-TR tienen una positividad similar para diagnosticar enfermedad por CMV en adultos chilenos con infección por VIH. El AVR es inapropiado como referencia por su baja positividad.

\section{Referencias bibliográficas}

1.- Luchsinger V, Luzoro A, Martínez M J. High seroprevalence of cytomegalovirus, herpes simplex type 1 virus and Epstein Barr virus infection among human immunodeficiency virus-infected adults. Rev Med Chile 2010; 138: 809-14.

2.- Landolfo S, Gariglio M, Gribaudo G, Lembo D. The human cytomegalovirus. Pharmacol Ther 2003; 98: 269-97.

3.- Sanbonmatsu S, Pérez M, Navarro J M. Infección por citomegalovirus humano. Enferm. Infecc. Microbiol. Clin. 2014, 32 (Supl 1): 1522.

4.- Griffiths P. CMV as a cofactor enhancing progression of AIDS. J Clin Virol 2006; 35 : 489-92.

5.- Steininger C, Puchhammer-Stöckl E, PopowKraupp T. Cytomegalovirus disease in the era of highly active antiretroviral therapy (HAART). J Clin Virol 2006; 37 (1): 1-9.

6.- Steininger C. Clinical relevance of cytomegalovirus infection in patients with disorders of the immune system. Clin Microbiol Infect 2007; 13 (10): 953-63.
7.- Ho M. The history of cytomegalovirus and its diseases. Med Microbiol Immunol 2002; 197: 65-73.

8.- Guía Clínica AUGE, MINSAL 2013.

9.- Razonable R. Management strategies for cytomegalovirus infection and disease in solid organ transplant recipients. Infect Dis Clin North Am 2013; 27: 317-42.

10.- Mhiri L, Kaabi B, Houimel M, Arrouji Z, Slim A. Comparison of pp65 antigenemia, quantitative PCR and DNA hybrid capture for detection of cytomegalovirus in transplant recipients and AIDS patients. J Virol Methods 2007; 143 (1): 23-8.

11.- Wolff M, Álvarez P, Flores I, Northland R, Wolff C. Evolución de mortalidad y estado actual de una población infectada por VIH controlada en un centro multiprofesional. Rev Med Chile 2006; 134: 581-8.

12.- Nagata N, Kobayakawa M, Shimbo T, Hoshimoto K, Yada T, Gotoda T, et al. Diagnostic value of antigenemia assay for cytomegalovirus gastrointestinal disease in immunocompromised patients. World $\mathrm{J}$ Gastroenterol 2011; 17 (9): 1185-91.

13.- Cariani E, Pollara C, Valloncini B, Perandin
F, Bonfanti C, Manca N. Relationship between pp65 antigenemia levels and realtime quantitative DNA PCR for human cytomegalovirus (HCMV) management in immunocompromised patients. BMC Infect Dis 2007; 7: 138-43.

14.- Shaade L, Kockelkorn P, Ritter K, Kleines M. Detection of cytomegalovirus DNA in human specimens by Light Cycler. J Clin Microbiol 2000; 38 (11): 4006-9.

15.- Chakraborty A, Mahapatra T, Mahapatra S, Ansari S, Siddhanta S, Banerjee S, et al. Distribution and determinants of cytomegalovirus induced end organ disease/s among people living with HIV/AIDS in a poor resource setting: observation from India. PLOS One $2015 ; 10$ (2): e 0117466

16.- Fica A, Cervera C, Pérez N, Marcos M A, Ramírez J, Linares L, et al. Immunohistochemically proven cytomegalovirus end-organ disease in solid organ transplant patients: clinical features and usefulness of conventional diagnostic tests. Transpl Infect Dis 2007; 9 (3): 203-10.

17.- Farfán M, Torres J, Vergara A, Donoso G, Alba A, Paris C, et al. Comparación de las 
técnicas de reacción de polimerasa en cadena en tiempo real y antigenemia para la detección de citomegalovirus en sangre de niños sometidos a trasplantes. Rev Chilena Infectol 2011; 28: 113-7.

18.- Ceballos M E, Vizcaya C, Pavez D, Cerda J, Martínez-Valdebenito C, Montecinos L, Ferrés M. Detección precoz de infección por citomegalovirus en pacientes sometidos a trasplante alogénico de precursores hematopoyéticos por reacción de polimerasa en cadena cuantitativa en tiempo real. Rev Chilena Infectol 2014; 31 (2): 153-9.

19.- Ljungman P, Griffiths P, Paya C. Definitions of cytomegalovirus infection and disease in transplant recipients. Clin Infect Dis 2002; 34 : 1094-7.

20.- Cerda J, Villarroel L. Evaluación de la concordancia inter-observador en investigación pediátrica: coeficiente de $\kappa$. Rev Chil Pediatr 2008; 79 (1): 54-8.

21.- Wattanamano P, Clayton J, Kopicko J, Kissinger P, Elliot S, Jarrott C, et al. Comparison of three assays for cytomegalovirus detection in AIDS patients at risk for retinitis. J Clin Microbiol 2000; 38 (2): 727-32.

22.- Hamada Y, Nagata N, Shimbo T, Igari T, Nakashima R, Asayama N, et al. Assessment of antigenemia assay for the diagnosis of cytomegalovirus gastrointestinal diseases in
HIV-infected patients. AIDS Patient Care and STDs 2013; 27 (7): 387-91.

23.- Hardie D, Korsman S, Hsiao N. Cytomegalovirus load in whole blood is more reliable for predicting and assessing CMV disease than pp65 antigenaemia. J Virol Methods 2013; 193: 166-8.

24.- El Amari E, Combescure C, Yerly S, Calmy A, Kaiser L, Hasse B, et al. Clinical relevance of cytomegalovirus viraemia. HIV Med 2011; 12 : 394-402.

25.- Durier N, Ananworanich J, Apornpong T, Ubolyam S, Kerr S, Mahanontharit A, et al. Cytomegalovirus viremia in Thai HIV-infected patients on antiretroviral therapy: prevalence and associated mortality. Clin Infect Dis 2013; 57 (1): 147-55.

26.- Fielding K, Koba A, Grant A, Charalambous S, Day J, Spak C, et al. Cytomegalovirus viremia as a risk factor for mortality prior to antiretroviral therapy among HIV-infected gold miners in South Africa. Plos One 2011; 6: e25571.

27.- Bek B, Boeckh M, Lepenies J, Bienek B, Arasteh K, Heise W, et al. High- level sensitivity of quantitative pp65 cytomegalovirus (CMV) antigenemia assay for diagnosis of CMV disease in AIDS patients and follow-up. J Clin Microbiol 1996, 34 (2): 457-9.

28.- Yoshida A, Hitomi S, Fukui T, Endo H,
Morisawa Y, Kazuyama Y, et al. Diagnosis and monitoring of human cytomegalovirus diseases in patients with human immunodeficiency virus infection by use of a real-time PCR assay. Clin Infect Dis 2001; 33: 1756-61.

29.- Gourlain K, Salmon D, Gault E, Leport C, Katlama C, Matheron S, et al. Quantitation of cytomegalovirus (CMV) DNA by realtime PCR for occurrence of CMV disease in HIV- infected patients receiving highly active antiretroviral therapy. J Med Virol 2003; 69: 401-7.

30.- Mengelle C, Pasquier C, Rostaing L, SandresSaune K, Puel J, Berges L, et al. Quantitation of human cytomegalovirus in recipients of solid organ transplants by real-time quantitative PCR and pp65 antigenemia. J Med Virol 2003, 69: 225-31.

31.- Gerna G, Percivalle E, Baldanti F, Sozzani S, Lanzarini P, Genini E, et al. Human cytomegalovirus replicates abortively in polymorphonuclear leukocytes after transfer from infected endothelial cells via transient microfusion events. J Virol 2000; 74 (12): 5629-38.

32.- Bego M G, St Jeor S. Human citomegalovirus infection of cells of hematopoietic origin: HCMV-induced immunosuppression, immune evasion, and latency. Experimental Hematol 2006; 34: 555-70. 